\title{
Dolbeault and J-Invariant Cohomologies on Almost Complex Manifolds
}

\author{
Lorenzo Sillari $^{1} \cdot$ Adriano Tomassini $^{2}$
}

Received: 24 March 2021 / Accepted: 23 August 2021 / Published online: 22 September 2021

(c) The Author(s) 2021

\begin{abstract}
In this paper we relate the cohomology of $J$-invariant forms to the Dolbeault cohomology of an almost complex manifold. We find necessary and sufficient condition for the inclusion of the former into the latter to be true up to isomorphism. We also extend some results obtained by J. Cirici and S. O. Wilson about the computation of the left-invariant cohomology of nilmanifolds to the setting of solvmanifolds. Several examples are given.
\end{abstract}

Keywords Almost complex manifold · Cohomology of Lie Algebra · Compact four-manifold · Dolbeault cohomology · Frölicher spectral sequence · Solvmanifold

Mathematics Subject Classification 32Q60 - 53C15

Communicated by F. Bracci.

This article is part of the topical collection "Higher Dimensional Geometric Function Theory and Hypercomplex Analysis" edited by Irene Sabadini, Michael Shapiro and Daniele Struppa.

Partially supported by the Project PRIN 2017 "Real and Complex Manifolds: Topology, Geometry and holomorphic dynamics" (Code 2017JZ2SW5) and by GNSAGA of INdAM. Data sharing not applicable to this article as no datasets were generated or analysed during the current study.

$\bowtie$ Adriano Tomassini

adriano.tomassini@unipr.it

Lorenzo Sillari

1sillari@sissa.it

1 Geometry and Mathematical Physics, Scuola Internazionale Superiore di Studi Avanzati (SISSA), Via Bonomea 265, 34136 Trieste, Italy

2 Dipartimento di Scienze Matematiche, Fisiche e Informatiche, Unità di Matematica e Informatica, Università degli Studi di Parma, Parco Area delle Scienze 53/A, 43124 Parma, Italy 


\section{Introduction}

Let $(M, J)$ be a $2 m$-dimensional almost complex manifold. Then the almost complex structure $J$ induces a bigrading on the bundle of differential forms on $M$. The exterior derivative $d$ acts on differential forms as the sum of four differential operators, $d=$ $\mu+\partial+\bar{\partial}+\bar{\mu}$.

The celebrated theorem of Newlander and Nirenberg states that $(M, J)$ admits the structure of complex manifold (i.e., $J$ is integrable) if and only if $N_{J}=0$, that is equivalent to $\mu=\bar{\mu}=0$. Consequently, in such a case $d=\partial+\bar{\partial}$. For complex manifolds it is classical and well established the theory of Dolbeault cohomology, obtained as the cohomology of the $\bar{\partial}$ operator. Another fundamental tool in the study of complex manifolds is the Hodge Theory for the $\bar{\partial}$ operator that, once fixed a Hermitian metric, establishes an isomorphism between the Dolbeault cohomology and the kernel of the Dolbeault Laplacian $\Delta_{\bar{\partial}}$. However for almost complex manifolds, the operator $\bar{\partial}$ is not cohomological and the Dolbeault cohomology cannot have the usual definition. It is natural to look for other cohomological theories to study geometric properties of almost complex manifolds. Motivated by the comparison between the J-tamed symplectic cone $\mathcal{K}_{J}^{t}$ and the $J$-compatible symplectic cone $\mathcal{K}_{J}^{c}$ of an almost complex manifold, defined as the projection in cohomology of the space of symplectic forms taming $J$, respectively calibrating $J, \mathrm{Li}$ and Zhang introduced in [13] two cohomology groups: the $J$-invariant cohomology groups, respectively $J$-anti-invariant cohomology groups, of an almost complex manifold $(M, J)$ denoted with $H^{+}$, respectively $H^{-}$, are formed by $2^{\text {nd }}$-de Rham classes represented by closed $J$-invariant forms, respectively $J$-anti-invariant forms, with respect to the natural action of $J$ on the space of 2-forms.

Such groups generalize the real Dolbeault cohomology classes in $H_{\bar{\partial}}^{1,1} \cap H_{d R}^{2}(\mathbb{R})$ and $\left(H_{\bar{\partial}}^{2,0}+H_{\bar{\partial}}^{0,2}\right) \cap H_{d R}^{2}(\mathbb{R})$ respectively. The focus is on whether the almost complex structure $J$ is $C^{\infty}$-pure, i.e., $H^{+} \cap H^{-}=\{0\}$ or $C^{\infty}$-full, i.e., $H_{d R}^{2}=H^{+}+H^{-}$. The problem is further studied in [10], where it is proved that any almost complex structure on a compact 4-manifold is $C^{\infty}$-pure and $C^{\infty}$-full, and in [11]. Such a result can be viewed as a sort of Hodge decomposition for 4-dimensional compact almost complex manifolds.

Recently Cirici and Wilson defined in [6] an analogous of Dolbeault cohomology for almost complex manifolds, that is also called Dolbeault cohomology. This definition of cohomology is based on the decomposition of $d$ and allows a development of a harmonic theory, at least in some favorable situation such as in [7] for the almost Kähler case (see also [17]). A Frölicher spectral sequence $E_{r}^{p, q}$ builds a bridge between the Dolbeault cohomology and the complex de Rham cohomology. In general, the computation of such groups is difficult, since they might not be finite-dimensional (cf. [8]). A special setting in which calculations can be performed is that of Lie Algebras. Such computations have a direct application in the study of the left-invariant Dolbeault cohomology of nilmanifolds, as showed in [6].

In this paper we study the relation between the complex cohomology group $H_{\mathbb{C}}^{+}$ of $J$-invariant complex forms and the Dolbeault cohomology group $H_{D o l}^{1,1}$ on almost complex manifolds. Next we extend some results obtained in [6] for nilmanifolds, to the case of solvmanifolds. More in details, since we have a characterization of $J$ - 
invariant 2-forms as real forms of complex bidegree $(1,1)$, it is natural to ask whether they belong or not to the Dolbeault cohomology groups, or at least if there exists an isomorphism between $H^{+}$and a subgroup of $H_{D o l}^{1,1}$. We relate $J$-invariant cohomology and Dolbeault cohomology, finding that the condition

$$
E_{1}^{0,1} \cong E_{2}^{0,1}
$$

is necessary and sufficient for the former cohomology group to be contained into the latter up to isomorphism (Theorem 4.2). Then given any solvmanifold endowed with a left-invariant almost complex structure, we prove that the left-invariant spectral sequence satisfies Serre duality at every page and that the left-invariant Dolbeault cohomology groups are isomorphic to the kernel of a suitable Laplacian (Theorem 6.1). We apply our results, and show how information on the left-invariant cohomologies can be used to deduce information on the non-left-invariant ones. Finally calculations of left-invariant spectral sequence and $J$-invariant cohomology are performed on almost complex manifolds and solvmanifolds endowed with a left-invariant almost complex structure to give concrete applications.

The paper is organized as follows. In Sect. 2 we briefly recall some basic definitions that will be used later on, and we introduce the notation. In Sect. 3 we resume the definition given by Cirici and Wilson of Dolbeault cohomology for almost complex manifolds. In particular, we focus on the spectral sequence arising from a Hodge filtration, and give an explicit description of it. Section 4 is devoted to the study of $J$-invariant cohomology and Dolbeault cohomology. We prove the results mentioned above, and investigate the behaviour of the necessary and sufficient condition under small deformations, showing with an example that it is not a closed property. Section 5 recalls the construction of the Dolbeault cohomology of Lie Algebras, while in Sect. 6 we prove the Serre duality for solvmanifolds and show a possible application of the results. Finally in Sect. 7 we collect various examples of Dolbeault cohomology and spectral sequences. Among them, we provide computations of the left-invariant spectral sequence on 4-dimensional solvmanifolds that do not admit any integrable almost complex structure. For such examples the Dolbeault cohomology theory for almost complex manifolds becomes the main tool to investigate their geometry.

\section{Preliminaries and Notation}

Let $(M, J)$ be an almost complex manifold of real dimension $2 m$, with $J$ an almost complex structure on the tangent bundle, i.e., $J \in E n d(T M)$ such that $J^{2}=-I d$. Denote by $A_{\mathbb{R}}^{*}$ (respectively $A_{\mathbb{C}}^{*}$ ) the algebras of real (respectively complex) differential forms on $M . J$ induces a bigrading on complex forms,

$$
A_{\mathbb{C}}^{k}=\bigoplus_{p+q=k} A_{\mathbb{C}}^{p, q} .
$$


On real $k$-forms, $\alpha \in A_{\mathbb{C}}^{k}, J$ induces a map still denoted by $J$ and defined by

$$
J \alpha\left(X_{1}, \ldots, X_{k}\right)=\alpha\left(J X_{1}, \ldots, J X_{k}\right)
$$

If $k$ is odd, $J^{2}=-I d$, while if $k$ is even, $J$ is an involution. In particular, $A_{\mathbb{R}}^{2}$ decomposes as

$$
A_{\mathbb{R}}^{2}=A_{\mathbb{R}}^{+}+A_{\mathbb{R}}^{-},
$$

where $A_{\mathbb{R}}^{+}$denotes the $J$-invariant forms and $A_{\mathbb{R}}^{-}$the $J$-anti-invariant forms. If we consider the bidegree induced on complex forms by $J$, it's easy to check that $A_{\mathbb{R}}^{+}$ consists of real forms in $A_{\mathbb{C}}^{1,1}$, while $A_{\mathbb{R}}^{-}$of real forms in $A_{\mathbb{C}}^{2,0}+A_{\mathbb{C}}^{0,2}$. We denote with $H_{d R}^{*}(\mathbb{R})$ (respectively $\left.H_{d R}^{*}(\mathbb{C})\right)$ the real (respectively complex) de Rham cohomology of $M$.

The de Rham cohomology groups consisting of $J$-invariant and $J$-anti-invariant forms were introduced in [13]. We shall use the notation of Drăghici et al. [11]. The $J$-invariant real cohomology group is

$$
H^{+}=\left\{[\alpha] \in H_{d R}^{2}(\mathbb{R}): \alpha \in A_{\mathbb{R}}^{+} \cap \operatorname{ker} d\right\},
$$

and the $J$-anti-invariant real cohomology group is

$$
H^{-}=\left\{[\alpha] \in H_{d R}^{2}(\mathbb{R}): \alpha \in A_{\mathbb{R}}^{-} \cap \operatorname{ker} d\right\} .
$$

In the following we will denote a $(p, q)$-form $\alpha$ with $\alpha^{p, q}$.

We call a solvmanifold the quotient of a connected, simply connected and solvable Lie Group $G$, by a discrete and co-compact subgroup $\Gamma$ of $G$. We denote it by $\Gamma \backslash G$. If $G$ is also nilpotent, we call $\Gamma \backslash G$ a nilmanifold.

\section{Dolbeault Cohomology and Spectral Sequences}

Let $(M, J)$ be an almost complex manifold of real dimension $2 m$. The exterior derivative decomposes as $d=\mu+\partial+\bar{\partial}+\bar{\mu}$, with bidegrees

$$
|\mu|=(2,-1), \quad|\partial|=(1,0), \quad|\bar{\partial}|=(0,1), \quad|\bar{\mu}|=(-1,2) .
$$

The almost complex structure $J$ is integrable if and only if $\bar{\mu} \equiv 0$. The equation $d^{2}=0$ gives the relations 


$$
\left\{\begin{array}{l}
\mu^{2}=0 \\
\mu \partial+\partial \mu=0 \\
\mu \bar{\partial}+\bar{\partial} \mu+\partial^{2}=0 \\
\mu \bar{\mu}+\partial \bar{\partial}+\bar{\partial} \partial+\bar{\mu} \mu=0 \\
\bar{\mu} \partial+\partial \bar{\mu}+\bar{\partial}^{2}=0 \\
\bar{\mu} \bar{\partial}+\bar{\partial} \bar{\mu}=0 \\
\bar{\mu}^{2}=0
\end{array}\right.
$$

Since $\bar{\mu}^{2}=0, \bar{\mu}$ is a well defined cohomological operator and its cohomology is the $\bar{\mu}$-cohomology

$$
H_{\bar{\mu}}^{p, q}=\frac{\operatorname{ker}\left(\bar{\mu}: A_{\mathbb{C}}^{p, q} \longrightarrow A_{\mathbb{C}}^{p-1, q+2}\right)}{\operatorname{Im}\left(\bar{\mu}: A_{\mathbb{C}}^{p+1, q-2} \longrightarrow A_{\mathbb{C}}^{p, q}\right)} .
$$

In general, $\bar{\partial}$ does not square to 0 on $M$, and its cohomology is not well defined. From the relation $\bar{\partial} \bar{\mu}+\bar{\mu} \bar{\partial}=0, \bar{\partial}$ is well defined on cohomology classes of $H_{\bar{\mu}}^{p, q}$, and thanks to $\bar{\partial}^{2}+\bar{\mu} \partial+\partial \bar{\mu}=0$, it squares to 0 , thus we can define [6, Definition 3.1] the Dolbeault cohomology of the almost complex manifold $(M, J)$ as the $\bar{\partial}$-cohomology of the $\bar{\mu}$-cohomology, i.e.,

$$
H_{D o l}^{p, q}=\frac{\operatorname{ker}\left(\bar{\partial}: H_{\bar{\mu}}^{p, q} \longrightarrow H_{\bar{\mu}}^{p, q+1}\right)}{\operatorname{Im}\left(\bar{\partial}: H_{\bar{\mu}}^{p, q-1} \longrightarrow H_{\bar{\mu}}^{p, q}\right)} .
$$

The cohomology groups are well defined and if $\bar{\mu}=0$, they coincide with the usual Dolbeault cohomology groups for complex manifolds. As in the complex case, the Dolbeault cohomology is induced by a filtration on differential forms and has an associated spectral sequence that coincides with the Frölicher spectral sequence of complex manifolds if $\bar{\mu}=0$. We recall here the construction: consider the filtration

$$
F^{p} A_{\mathbb{C}}^{k}=A_{\mathbb{C}}^{p, q} \cap \operatorname{ker} \bar{\mu} \oplus \bigoplus_{j \geq p+1} A_{\mathbb{C}}^{j, k-j} .
$$

The filtration is bounded by 0 from below and by $m$ from above. With a shift of indexing, the filtration coincides with the filtration of a suitable multicomplex endowed with 4 differentials, and the two spectral sequences are isomorphic, up to taking pages shifted by one step. Denote by

$$
E_{r}^{p, q}, \quad p, q=0, \ldots, m, r \geq 1,
$$

the pages of the sequence. Then $E_{1}^{p, q} \cong H_{D o l}^{p, q}$ and the $(r+1)$-th page is the cohomology of the previous one, 


$$
E_{r+1}^{p, q} \cong \frac{\operatorname{ker}\left(d_{r}: E_{r}^{p, q} \longrightarrow E_{r}^{p+r, q-r+1}\right)}{\operatorname{Im}\left(d_{r}: E_{r}^{p-r, q+r-1} \longrightarrow E_{r}^{p, q}\right)},
$$

with respect to the differential $d_{r},\left|d_{r}\right|=(r,-r+1)$. An explicit description up to isomorphism of the differential $d_{r}$ and of the pages of the spectral sequence is given in [14] for a general multicomplex, and was first described in [9] for the Frölicher spectral sequence of a complex manifold. For page 1, we have

$$
E_{1}^{p, q} \cong \frac{\left\{\alpha \in A_{\mathbb{C}}^{p, q} \cap \operatorname{ker} \bar{\mu}: \bar{\partial} \alpha \in \operatorname{Im} \bar{\mu}\right\}}{\left\{\eta \in A_{\mathbb{C}}^{p, q}: \eta=\bar{\mu} a+\bar{\partial} b \text { and } \bar{\mu} b=0\right\}},
$$

and if $\bar{\partial} \alpha=\bar{\mu} \varphi$, then

$$
d_{1}[\alpha]_{E_{1}}=[\partial \alpha-\bar{\partial} \varphi]_{E_{1}} .
$$

In general, we have an isomorphism

$$
E_{r}^{p, q} \cong \frac{X_{r}^{p, q}}{Y_{r}^{p, q}}
$$

where

$$
\begin{aligned}
X_{r}^{p, q} & =\left\{\alpha^{p, q} \in A_{\mathbb{C}}^{p, q}: \text { there exist } \alpha^{p+j, q-j}, j=1, \ldots r,\right. \text { satisfying } \\
0 & \left.=\mu \alpha^{p+k, q-k}+\partial \alpha^{p+k+1, q-k-1}+\bar{\partial} \alpha^{p+k+2, q-k-2}+\bar{\mu} \alpha^{p+k+3, q-k-3}, \quad k \in \mathbb{Z}\right\}
\end{aligned}
$$

where the equation has to be read with $\alpha^{p+k, q-k}=0$ if $k$ is not $0,1, \ldots, r$, and

$$
\begin{aligned}
Y_{r}^{p, q}= & \left\{\eta^{p, q} \in A_{C}^{p, q}: \text { there exist } \eta^{p-j, q+j-1}, j=-1,0, \ldots, r-1\right. \text { satisfying } \\
\eta^{p, q}= & \mu \eta^{p-2, q+1}+\partial \eta^{p-1, q}+\bar{\partial} \eta^{p, q-1}+\bar{\mu} \eta^{p+1, q-2} \\
0= & \mu \eta^{p-k, q+k-1}+\partial \eta^{p-k+1, q+k-2}+\bar{\partial} \eta^{p-k+2, q+k-3} \\
& \left.+\bar{\mu} \eta^{p-k+3, q+k-4} \quad k=3, \ldots, r-1\right\} .
\end{aligned}
$$

We say that the spectral sequence degenerates at page $r$, for bidegree $(p, q)$, and write $E_{r}^{p, q} \cong E_{\infty}^{p, q}$, if

$$
E_{r}^{p, q} \cong E_{j}^{p, q} \quad \forall j \geq r
$$

The spectral sequence degenerates at page $r$ if it degenerates at page $r$ for all bidegrees.

At the $E_{\infty}$ page, the degeneration of the spectral sequence induces a bigrading on the de Rham cohomology of the almost complex manifold. In particular, $E_{\infty}^{p, q}$ consists of cohomology classes in $H_{d R}^{p+q}(\mathbb{C})$ that admit a complex representative of bidegree $(p, q)$. 


\section{Inclusion of $J$-Invariant Cohomology into Dolbeault Cohomology}

Denote by $H_{\mathbb{C}}^{+}=H^{+} \otimes \mathbb{C}$ the complexified of the $J$-invariant cohomology group. We are going to study under which conditions $H_{\mathbb{C}}^{+}$is isomorphic to a subgroup of $H_{D o l}^{1,1}$ through the isomorphism of (3.9) between the Frölicher spectral sequence and the quotients $X_{r}^{p, q} / Y_{r}^{p, q}$.

The results are stated in Theorem 4.2, that gives a characterization valid in the almost complex case. At the end, we briefly investigate the stability of the condition found in the theorem under small deformations (in the integrable case).

For almost complex manifolds of any dimension, $H_{\mathbb{C}}^{+}$consists of complex de Rham cohomology classes in $H_{d R}^{2}(\mathbb{C})$ that admit a representative of type $(1,1)$ (cf. [10], Lemma 2.11).

We begin considering the map in cohomologies induced by the identity on representatives and show that it is never well defined.

Proposition 4.1 Let $(M, J)$ be an almost complex manifold. The map

$$
\begin{aligned}
& H_{\mathbb{C}}^{+} \longrightarrow H_{D o l}^{1,1} \\
& {\left[\alpha^{1,1}\right]^{+} \longmapsto\left[\alpha^{1,1}\right]_{\text {Dol }}}
\end{aligned}
$$

is not well defined.

Proof The cohomology class $\left[\alpha^{1,1}\right]^{+}$is written as

$$
\left[\alpha^{1,1}\right]^{+}=\left\{\alpha^{1,1}+d\left(\beta^{1,0}+\beta^{0,1}\right): d \alpha^{1,1}=0\right\} .
$$

In particular, $\bar{\partial} \alpha^{1,1}=\bar{\mu} \alpha^{1,1}=0$. This implies that there exists a cohomology class in $H_{D o l}^{1,1}$ given by $\left[\alpha^{1,1}\right]_{D o l}$. However the definition of the map is not independent of the representative chosen in $\left[\alpha^{1,1}\right]^{+}$. We show now that this independence can never occur. Checking independence on the representative reduces to check that

$$
[0]^{+} \subseteq[0]_{D o l} \in H_{D o l}^{1,1}
$$

Assume that this is the case, i.e. that

$$
d\left(\beta^{1,0}+\beta^{0,1}\right) \in[0]_{D o l} \quad \forall \beta^{1,0} \in A_{\mathbb{C}}^{1,0}, \beta^{0,1} \in A_{\mathbb{C}}^{0,1} .
$$

Taking $\beta^{0,1}=0$, we have that

$$
\left[d \beta^{1,0}\right]_{D o l}=\left[\partial \beta^{1,0}+\bar{\partial} \beta^{1,0}+\bar{\mu} \beta^{1,0}\right]_{D o l}=\left[\partial \beta^{1,0}+\bar{\partial} \beta^{1,0}\right]_{D o l} .
$$

Since the terms $\partial \beta^{1,0}$ and $\bar{\partial} \beta^{1,0}$ have different bidegree, they must be in the trivial class separately. The first one is trivial if and only if $\partial \beta^{1,0}=0$ for every $\beta^{1,0} \in A_{\mathbb{C}}^{1,0}$. In other words $\partial$ must vanish on $(1,0)$-forms. The second one is trivial if and only if $\bar{\mu}$ vanishes on $(1,0)$-forms, i.e. if the almost complex structure $J$ is integrable. 
This proves that if the inclusion is well defined $M$ must be a complex manifold with vanishing $\partial$ on $(1,0)$-forms. This is not the case even on complex tori.

In order to obtain an affirmative result, we must consider the inclusion up to isomorphism. In particular we focus on the isomorphism (3.9), that allows to use the explicit description of the pages of the spectral sequence recalled in (3.10) and (3.11).

At bidegree $(1,1)$, the $r$-th page of the spectral sequence is $E_{r}^{1,1} \cong X_{r}^{1,1} / Y_{r}^{1,1}$, where

$$
\begin{aligned}
& X_{1}^{1,1}=\left\{\alpha^{1,1}: 0=\bar{\mu} \alpha^{1,1}=\bar{\partial} \alpha^{1,1}+\bar{\mu} \alpha^{2,0}\right\}, \\
& X_{2}^{1,1}=\left\{\alpha^{1,1}: 0=\bar{\mu} \alpha^{1,1}=\bar{\partial} \alpha^{1,1}+\bar{\mu} \alpha^{2,0}=\partial \alpha^{1,1}+\bar{\partial} \alpha^{2,0}\right\}, \\
& X_{3}^{1,1}=\left\{\alpha^{1,1}: 0=\bar{\mu} \alpha^{1,1}=\bar{\partial} \alpha^{1,1}+\bar{\mu} \alpha^{2,0}=\partial \alpha^{1,1}+\bar{\partial} \alpha^{2,0}=\mu \alpha^{1,1}+\partial \alpha^{2,0}\right\}, \\
& Y_{1}^{1,1}=\left\{\eta^{1,1}=\bar{\partial} \eta^{1,0}, \bar{\mu} \eta^{1,0}=0\right\}, \\
& Y_{2}^{1,1}=Y_{3}^{1,1}=\left\{\eta^{1,1}=\bar{\partial} \eta^{1,0}+\partial \eta^{0,1}, \bar{\mu} \eta^{1,0}+\bar{\partial} \eta^{0,1}=0\right\} .
\end{aligned}
$$

Note that the spectral sequence at bidegree $(1,1)$ degenerates at most at page 3 independently of the dimension of the manifold. In fact $\left|d_{r}\right|=(r,-r+1)$, then

$$
0 \stackrel{d_{r}}{\longrightarrow} E_{r}^{1,1} \stackrel{d_{r}}{\longrightarrow} 0,
$$

if $r \geq 3$, and $E_{\infty}^{1,1}=E_{3}^{1,1}$. If $m=2$, then it degenerates at the second page. Indeed, if $M$ is compact, $J$ is integrable and $m=2$ (i.e., if $M$ is a compact complex surface) or if $M$ is a compact Kähler manifold of any dimension, it degenerates at the first page and the Dolbeault cohomology group at bidegree $(1,1)$ is isomorphic to the complexified of $\mathrm{H}^{+}$(cf. [13], Theorem 2.16).

If $J$ is integrable, we simply have

$$
\begin{aligned}
& X_{1}^{1,1}=\left\{\alpha^{1,1}: 0=\bar{\partial} \alpha^{1,1}\right\}, \\
& X_{2}^{1,1}=\left\{\alpha^{1,1}: 0=\bar{\partial} \alpha^{1,1}=\partial \alpha^{1,1}+\bar{\partial} \alpha^{2,0}\right\}, \\
& X_{3}^{1,1}=\left\{\alpha^{1,1}: 0=\bar{\partial} \alpha^{1,1}=\partial \alpha^{1,1}+\bar{\partial} \alpha^{2,0}=\partial \alpha^{2,0}\right\}, \\
& Y_{1}^{1,1}=\left\{\eta^{1,1}=\bar{\partial} \eta^{1,0}\right\} \\
& Y_{2}^{1,1}=Y_{3}^{1,1}=\left\{\eta^{1,1}=\bar{\partial} \eta^{1,0}+\partial \eta^{0,1}, \bar{\partial} \eta^{0,1}=0\right\} .
\end{aligned}
$$

Before stating the theorem, we make a consideration on the spectral sequence at bidegree $(0,1)$. We have

$$
0 \stackrel{d_{3}}{\longrightarrow} E_{3}^{0,1} \stackrel{d_{3}}{\longrightarrow} 0,
$$

so that $E_{3}^{0,1}=E_{r}^{0,1}$ for all $r \geq 3$, but $E_{1}^{0,1}=E_{2}^{0,1}$ does not imply $E_{2}^{0,1}=E_{3}^{0,1}$, since in general

$$
E_{2}^{0,1} \stackrel{d_{2}}{\longrightarrow} E_{2}^{2,0}
$$


does not vanish.

In what follows we establish a necessary and sufficient condition in order to have an inclusion up to isomorphism of the complexified $J$-invariant cohomology into the Dolbeault cohomology.

Theorem 4.2 Let $(M, J)$ be an almost complex manifold. Denote by $\varphi$ the isomorphism $E_{r}^{p, q} \cong X_{r}^{p, q} / Y_{r}^{p, q}$. Then the following conditions are equivalent

(i) $\varphi\left(H_{\mathbb{C}}^{+}\right) \subseteq \varphi\left(H_{D o l}^{1,1}\right)$;

(ii) $E_{1}^{0,1} \stackrel{\varphi}{\cong} E_{2}^{0,1}$.

If (i) [or (ii)] holds, then the inclusion is injective.

Proof Using the isomorphism $\varphi$, (i) is equivalent to prove that

$$
\frac{X_{3}^{1,1}}{Y_{3}^{1,1}} \subseteq \frac{X_{1}^{1,1}}{Y_{1}^{1,1}},
$$

while (ii) is equivalent to

$$
\frac{X_{1}^{0,1}}{Y_{1}^{0,1}}=\frac{X_{2}^{0,1}}{Y_{2}^{0,1}} .
$$

$X_{r}^{p, q}$ and $Y_{r}^{p, q}$ satisfy by definition the following chain of inclusions:

$$
Y_{1}^{p, q} \subseteq Y_{2}^{p, q} \subseteq \cdots \subseteq Y_{\infty}^{p, q} \subseteq X_{\infty}^{p, q} \subseteq \cdots \subseteq X_{2}^{p, q} \subseteq X_{1}^{p, q}
$$

For $(p, q)=(1,1)$, using that $Y_{2}^{1,1}=Y_{3}^{1,1}$, this reduces to

$$
Y_{1}^{p, q} \subseteq Y_{2}^{p, q} \subseteq X_{3}^{p, q} \subseteq X_{2}^{p, q} \subseteq X_{1}^{p, q}
$$

We begin by proving that condition (i) holds if and only if $Y_{1}^{1,1}=Y_{2}^{1,1}$. In fact if (i) is satisfied, we consider elements in the quotient $X_{3}^{1,1} / Y_{2}^{1,1}$ of the form $0+Y_{2}^{1,1}$. Such elements belong to the quotient $X_{1}^{1,1} / Y_{1}^{1,1}$ if $Y_{2}^{1,1} \subseteq Y_{1}^{1,1}$. Since $Y_{1}^{1,1}$ is always a subset of $Y_{2}^{1,1}$, we have $Y_{2}^{1,1}=Y_{1}^{1,1}$. Conversely, if $Y_{2}^{1,1}=Y_{1}^{1,1}$, then (i) reduces to

$$
\frac{X_{3}^{1,1}}{Y_{1}^{1,1}} \subseteq \frac{X_{1}^{1,1}}{Y_{1}^{1,1}},
$$

that is true thanks to the general relation $X_{3}^{1,1} \subseteq X_{1}^{1,1}$.

We prove now equivalence with (ii). The differential $d_{1}$ of the spectral sequence computed on $E_{1}^{0,1}$ is

$$
d_{1}\left[\eta^{0,1}\right]_{1}=\left[\bar{\partial} \eta^{1,0}+\partial \eta^{0,1}\right]_{1}
$$


where $\eta^{1,0}$ is any $(1,0)$-form such that $\bar{\mu} \eta^{1,0}+\bar{\partial} \eta^{0,1}=0$.

Writing the explicit expression of $Y_{2}^{1,1}$, we have that $Y_{2}^{1,1} \subseteq Y_{1}^{1,1}$ if and only if

$$
\text { for all } \eta^{1,0}, \eta^{0,1} \text { satisfying } \bar{\mu} \eta^{1,0}+\bar{\partial} \eta^{0,1}=0 \text {, }
$$

there exists $\gamma^{1,0}$ such that $\bar{\partial} \eta^{1,0}+\partial \eta^{0,1}=\bar{\partial} \gamma^{1,0}$, with $\bar{\mu} \gamma^{1,0}=0$, i.e.

$$
E_{1}^{0,1} \stackrel{d_{1}=0}{\longrightarrow} E_{1}^{1,1}
$$

or, equivalently, $X_{1}^{0,1}=X_{2}^{0,1}$. This gives the desired isomorphism since $E_{2}^{0,1} \cong$ ker $d_{1} \cap E_{1}^{0,1} \cong E_{1}^{0,1}$. Injectivity follows immediately from

$$
Y_{1}^{1,1} \cap X_{3}^{1,1} \subseteq Y_{1}^{1,1}=Y_{3}^{1,1}
$$

In Example 7.1, we will show in a concrete case how the inclusion is not well defined if the condition $E_{1}^{0,1} \cong E_{2}^{0,1}$ is not satisfied, even in the favorable case of a complex manifold. In Remark 7.3, we will note that Example 7.2 shows how the same occurs for the left-invariant Dolbeault cohomology on an almost complex manifold (the left-invariant cohomology will be introduced in the following sections).

For the inclusion up to isomorphism, the key condition is the isomorphism between two terms of the spectral sequence

$$
E_{1}^{0,1} \cong E_{2}^{0,1},
$$

thus we find meaningful considering the openness and closedness of $(*)$ under small deformations of complex structure.

Let $M$ be a compact complex manifold (so that the $E_{r}^{p, q}$ are finite dimensional) and $\left\{J_{t}\right\}$ a deformation of complex structures on $M$, with small $t \in \mathbb{C}$. An easy calculation shows that if we assume $(*)$ for $t=0$, the function

$$
e_{2}^{0,1}(t)=\operatorname{dim} E_{2}^{0,1}(t),
$$

is upper semicontinuous with respect to $t$. Indeed we have upper semicontinuity of $h^{p, q}(t)=\operatorname{dim} H_{\bar{\partial}}^{p, q}(t)$. Then

$$
e_{2}^{0,1}(t) \leq h^{0,1}(t) \leq h^{0,1}(0)=e_{2}^{0,1}(0) \text {. }
$$

We ask the following

Question let $\left(M, J_{0}\right)$ be a compact complex manifold. Is condition (*) stable under small deformations of the complex structure $J_{0}$ ? 
In all the examples for which we performed computations, the stability is satisfied.

On the other side, as a consequence of Example 7.1, we have that $(*)$ is not a closed condition even if we assume the integrability of $J$. More precisely, the example shows the following proposition.

Proposition 4.3 There exist complex manifolds $(M, J)$ such that

(i) the spectral sequence satisfies

$$
E_{1}^{0,1} \neq E_{2}^{0,1}
$$

(ii) there are curves of complex structures $\left\{J_{t}\right\}$ satysfying $J_{0}=J$ and

$$
E_{1}^{0,1}(t) \cong E_{2}^{0,1}(t)
$$

for all small $t \neq 0$.

The same happens at the level of the left-invariant spectral sequence for almost complex manifold as shown in Example 7.4.

\section{Dolbeault Cohomology of Lie Algebras}

Let $\mathfrak{g}$ be a real Lie Algebra of dimension $2 m$ and $J$ a complex structure on the vector space $\mathfrak{g}$. We call $J$ an almost complex structure on the Lie Algebra $\mathfrak{g}$. Consider the Chevalley-Eilenberg complex of $\mathfrak{g},\left(A_{\mathfrak{g}}^{*}, d\right)$. Recall that the differential is defined as the dual of the Lie bracket $[\cdot, \cdot]$ for 1 -forms, and extended as a derivation to all forms. $J$ induces a bidegree on the complexified of the Chevalley-Eilenberg complex,

$$
A_{\mathfrak{g}^{\mathbb{C}}}^{k}=\bigoplus_{p+q=k} A_{\mathfrak{g}^{\mathbb{C}}}^{p, q}
$$

The Dolbeault cohomology of the Lie Algebra $\mathfrak{g}$ is the $\bar{\partial}$-cohomology of the $\bar{\mu}$ cohomology groups,

$$
H_{D o l}^{p, q}(\mathfrak{g})=H^{q}\left(H_{\bar{\mu}}^{p, *}(\mathfrak{g}), \bar{\partial}\right)
$$

and the spectral sequence $\left\{E_{r}^{*, *}(\mathfrak{g})\right\}_{r \in \mathbb{N}}$ associated to the Hodge filtration

$$
F^{p} A_{\mathfrak{g}^{\mathbb{C}}}^{k}=A_{\mathfrak{g}^{\mathbb{C}}}^{p, q} \cap \operatorname{ker} \bar{\mu} \oplus \bigoplus_{j \geq p+1} A_{\mathfrak{g}^{\mathbb{C}}}^{j, k-j}
$$

is the spectral sequence of $\mathfrak{g}$. In the setting of Lie Algebras, it's possible to compute easily the cohomology as a matter of linear algebra and all the spaces are finite dimensional. We set

$$
b_{\mathfrak{g}}^{k}=\operatorname{dim}_{\mathbb{C}} H_{d R}^{k}(\mathfrak{g}, \mathbb{C})
$$


and

$$
h_{\mathfrak{g}}^{p, q}=\operatorname{dim}_{\mathbb{C}} H_{D o l}^{p, q}(\mathfrak{g}) .
$$

As a consequence of the existence of the spectral sequence, we have Frölicher inequalities (cf. [12]) for the almost complex case.

Theorem 5.1 ([6], Proposition 5.1) Let $\mathfrak{g}$ be a real Lie Algebra, dim $\mathfrak{g}=2 m$, and $J$ an almost complex structure on $\mathfrak{g}$. Then

$$
b_{g}^{k} \leq \sum_{p+q=k} h_{\mathfrak{g}}^{p, q} .
$$

Denote by $\chi(\mathfrak{g})=\sum_{k}(-1)^{k} b^{k}$ the Euler characteristic of $\mathfrak{g}$. Then

$$
\chi(\mathfrak{g})=\sum_{p, q}(-1)^{p+q} h_{\mathfrak{g}}^{p, q} .
$$

Consider now a $J$-compatible inner product $\langle\cdot, \cdot\rangle$ on $\mathfrak{g}$. It is possible to develop a harmonic theory for differential operators that makes easier some computations of cohomology groups. The Hodge * operator is defined as usual by the relation

$$
\langle\varphi, \eta\rangle V o l=\varphi \wedge * \bar{\eta},
$$

with $V o l$ denoting the volume form in $A_{\mathfrak{g}^{\mathbb{C}}}^{2 m}$, and $\varphi, \eta \in A_{\mathfrak{g}^{\mathbb{C}}}^{p, q}$. Taken $\delta$ among $d, \mu, \partial$, $\bar{\partial}, \bar{\mu}$, the formal adjoint of $\delta$ is the operator

$$
\delta^{*}=-* \bar{\delta} *
$$

The $\delta$-Laplacian is defined as

$$
\Delta_{\delta}=\delta \delta^{*}+\delta^{*} \delta
$$

and the space of $\delta$-harmonic $(p, q)$-forms is

$$
\mathcal{H}_{\delta}^{p, q}=A_{\mathfrak{g}^{\mathbb{C}}}^{p, q} \cap \operatorname{ker} \Delta_{\delta}
$$

On a Lie Algebra, the above spaces are always finite dimensional.

The operator $\bar{\mu}^{*}$ is the adjoint of $\bar{\mu}$ with respect to $\langle\cdot, \cdot\rangle$, and $A_{\mathfrak{g}^{\mathbb{C}}}^{p, q}$ admits a Hodge decomposition

$$
A_{\mathfrak{g}^{\mathbb{C}}}^{p, q}=\mathcal{H}_{\bar{\mu}}^{p, q} \oplus \bar{\mu}\left(A_{\mathfrak{g}^{\mathbb{C}}}^{p+1, q-2}\right) \oplus \bar{\mu}^{*}\left(A_{\mathfrak{g}^{\mathbb{C}}}^{p-1, q+2}\right) .
$$

In particular, cohomology classes in $H_{\bar{\mu}}^{p, q}(\mathfrak{g})$ admit a $\bar{\mu}$-harmonic representative. For a Lie Algebra, the Dolbeault cohomology can also be obtained as the cohomology of the operator $\bar{\partial}_{\bar{\mu}}$, defined on $\bar{\mu}$-harmonic $(p, q)$-forms as 


$$
\bar{\partial}_{\bar{\mu}}(\varphi)=\mathcal{H}_{\bar{\mu}}(\bar{\partial} \varphi)
$$

where we denoted with $\mathcal{H}_{\bar{\mu}}$ the projection on $\bar{\mu}$-harmonic forms. It can be checked that $\bar{\partial}_{\bar{\mu}}$ is a cohomological operator and

$$
H_{D o l}^{p, q}(\mathfrak{g}) \cong \frac{\operatorname{ker}\left(\bar{\partial}_{\bar{\mu}}: \mathcal{H}_{\bar{\mu}}^{p, q} \rightarrow \mathcal{H}_{\bar{\mu}}^{p, q+1}\right)}{\operatorname{Im}\left(\bar{\partial}_{\bar{\mu}}: \mathcal{H}_{\bar{\mu}}^{p, q-1} \rightarrow \mathcal{H}_{\bar{\mu}}^{p, q}\right)} .
$$

Taking the adjoint $\bar{\partial}_{\bar{\mu}}^{*}:=\mathcal{H}_{\bar{\mu}} \circ \bar{\partial}^{*}$, we can consider the associated Laplacian

$$
\Delta_{\bar{\partial}_{\bar{\mu}}}=\bar{\partial}_{\bar{\mu}}^{*} \bar{\partial}_{\bar{\mu}}+\bar{\partial}_{\bar{\mu}} \bar{\partial}_{\bar{\mu}}^{*} .
$$

The space of $\bar{\partial}_{\bar{\mu}}$-harmonic forms is

$$
\mathcal{H}_{\bar{\partial}_{\bar{\mu}}}^{p, q}=\mathcal{H}_{\bar{\mu}}^{p, q} \cap \operatorname{ker} \Delta_{\bar{\partial}_{\bar{\mu}}} .
$$

The main obstruction to using $\bar{\partial}_{\bar{\mu}}$ to study the Dolbeault cohomology, lies in the fact that in general, $\bar{\partial}_{\bar{\mu}}^{*}$ is not the metric adjoint of $\bar{\partial} \bar{\mu}$. However, this is the case in some favorable situation, in particular for compact Lie Groups or for the left-invariant cohomology of solvmanifolds.

\section{Dolbeault Cohomology of Solvmanifolds Endowed with a Left-Invariant Almost Complex Structure}

In this section we extend results obtained by J. Cirici and S. O. Wilson in [6] for nilmanifolds, to the case of solvmanifolds, showing that the left-invariant Dolbeault cohomology always satisfies Serre duality and is described by $\bar{\partial}_{\bar{\mu}}$. Finally, we show how Theorem 4.2 can be used, together with the left-invariant cohomology, to recover informations on the (non-left-invariant) Dolbeault cohomology.

Let $M=\Gamma \backslash G$ be a solvmanifold. Consider the three graded algebra:

- $A_{\mathfrak{g}}^{*}$, algebra of differential forms on $\mathfrak{g}$;

- ${ }^{L} A^{*}(M)$, algebra of left-invariant forms on $M$;

- $A^{*}(M)$, algebra of differential forms on $M$.

There is always an isomorphism

$$
A_{\mathfrak{g}}^{*} \cong{ }^{L} A^{*}(M)
$$

and an inclusion

$$
{ }^{L} A^{*}(M) \hookrightarrow A^{*}(M)
$$

Both clearly extend to the complexified version of the algebras. The isomorphism of the Chevalley-Eilenberg complex with left-invariant forms, induces an isomorphism 
of the de Rham cohomology of $\mathfrak{g}$ and the left-invariant de Rham cohomology of $M$ (i.e., the de Rham cohomology of left-invariant forms),

$$
H_{d R}^{*}(\mathfrak{g} ; \mathbb{C}) \cong{ }^{L} H_{d R}^{*}(M ; \mathbb{C})
$$

but in general the inclusion is not a quasi-isomorphism, and the left-invariant de Rham cohomology is not isomorphic to the de Rham cohomology of $M$. However, that happens in some favorable case. In particular, if $M$ itself is a compact Lie Group, or if $G$ is completely solvable, then

$$
H_{d R}^{*}(\mathfrak{g} ; \mathbb{C}) \cong H_{d R}^{*}(M ; \mathbb{C})
$$

An almost complex structure $\tilde{J}$ defined on $\mathfrak{g}$ induces in a natural way an almost complex structure $J$ on the solvmanifold $M$. We call such a $J$, a left-invariant almost complex structure on $M . \tilde{J}$ is integrable if and only if $J$ is integrable as an almost complex structure on $M$.

The left-invariant Dolbeault cohomology of $M$ is defined as the Dolbeault cohomology of the complexified Lie Algebra,

$$
{ }^{L} H_{D o l}^{p, q}(M)=H_{D o l}^{p, q}(\mathfrak{g}),
$$

and the left-invariant spectral sequence of $M$ as the spectral sequence associated to the Dolbeault cohomology of $\mathfrak{g}$,

$$
{ }^{L} E_{r}^{*, *}=E_{r}^{*, *}(\mathfrak{g})
$$

One can consider also the non-left-invariant Dolbeault cohomology associated to $J$. It is not known if this cohomology coincides with the left-invariant one, even in the case when (6.4) holds, but this is conjectured to be true for nilmanifolds and integrable almost complex structures (cf. [16]).

We want to prove the following theorem.

Theorem 6.1 Let $M=\Gamma \backslash G$ be a solvmanifold. Then for all $(p, q)$, its left-invariant Dolbeault cohomology is obtained as $\bar{\partial}_{\bar{\mu}}$-harmonic left-invariant forms,

$$
{ }^{L} H_{D o l}^{p, q}(M) \cong{ }^{L} \mathcal{H}_{\bar{\partial}_{\bar{\mu}}}^{p, q} .
$$

The left-invariant spectral sequence satisfies Serre duality at every page

$$
{ }^{L} E_{r}^{p, q} \cong{ }^{L} E_{r}^{m-p, m-q}, \quad \forall r \geq 1 .
$$

Before giving the proof of the theorem, we make some preliminary observation and state some useful Lemma. 
The study of left-invariant cohomology is made easy if $\bar{\partial}_{\bar{\mu}}^{*}$ is the metric adjoint of $\bar{\partial}_{\bar{\mu}}$. A sufficient condition for this to happen (cf. [6], Lemma 5.2) is

$$
\partial \equiv 0 \quad \text { on } A_{\mathfrak{g}^{\mathbb{C}}}^{m-1, m} .
$$

Equivalent conditions to (6.9) are

$$
d \equiv 0 \text { on } A_{\mathfrak{g}^{\mathbb{C}}}^{2 m-1}
$$

and

$$
H_{d R}^{2 m}(\mathfrak{g} ; \mathbb{C}) \cong \mathbb{C}
$$

We recall now some consequence of (6.10), that will be used to prove Theorem 6.1. The fact that $\bar{\partial}_{\bar{\mu}}^{*}$ is adjoint of $\bar{\partial}_{\bar{\mu}}$, allows to use harmonic theory to establish an isomorphism from $\overline{\partial_{\bar{\mu}}}$-harmonic forms to the Dolbeault cohomology of $\mathfrak{g}$, (and consequently to the left-invariant cohomology of $M$ ).

Proposition 6.2 ([6], Theorem 5.4) Let $\mathfrak{g}$ be a Lie Algebra and $H_{d R}^{2 m}(\mathfrak{g} ; \mathbb{C}) \cong \mathbb{C}$. Then

$$
\mathcal{H}_{\bar{\mu}}^{p, q}=\mathcal{H}_{\bar{\partial}}^{p, q} \oplus \bar{\partial}_{\bar{\mu}}\left(\mathcal{H}_{\bar{\mu}}^{p, q-1}\right) \oplus \bar{\partial}_{\bar{\mu}}^{*}\left(\mathcal{H}_{\bar{\mu}}^{p, q+1}\right)
$$

and $\bar{\partial}_{\bar{\mu}}$-harmonic forms are isomorphic to the Dolbeault cohomology of $\mathfrak{g}$,

$$
H_{D o l}^{p, q}(\mathfrak{g}) \cong \mathcal{H}_{\bar{\partial}_{\bar{\mu}}}^{p, q}
$$

The Hodge $*$ operator and conjugation give, with the usual argumentation, Serre duality for $\mathcal{H}_{\bar{\partial}}^{p}, q$, and via the above isomorphism, the first page of the left-invariant spectral sequence also satisfies Serre duality.

Proposition 6.3 ([6], Corollary 5.5) Let $\mathfrak{g}$ be a Lie Algebra and $H^{2 m}(\mathfrak{g} ; \mathbb{C}) \cong \mathbb{C}$. Then

$$
H_{D o l}^{p, q}(\mathfrak{g}) \cong H_{D o l}^{m-p, m-q}(\mathfrak{g})
$$

For the sake of completeness, we recall the proof of the following well known result.

Lemma 6.4 Let $\mathfrak{g}$ be a unimodular Lie Algebra, $\operatorname{dim}_{\mathbb{R}} \mathfrak{g}=2 m$. Then $d \equiv 0$ on $A_{\mathfrak{g}^{\mathbb{C}}}^{2 m-1}$.

Proof Fix an inner product $\langle\cdot, \cdot\rangle$ on $\mathfrak{g}$. Let $\left\{e_{j}\right\}_{j=1}^{2 m}$ be an ortonormal basis of $\mathfrak{g}$ with respect to the inner product and $\left\{\phi^{j}\right\}_{j=1}^{2 m}$ its dual basis. Set

$$
\left[e_{j}, e_{k}\right]=\sum_{l} C_{j k}^{l} e_{l}, \quad C_{j k}^{l}+C_{k j}^{l}=0
$$


The differential on 1-forms is determined by the structure constants

$$
d \phi^{l}=-\frac{1}{2} \sum_{j, k} C_{j k}^{l} \phi^{j} \wedge \phi^{k} .
$$

A basis of $(2 m-1)$-forms is given by $\left\{\phi^{1} \wedge \cdots \wedge \hat{\phi}^{j} \wedge \cdots \wedge \phi^{2 m}\right\}_{j=1}^{2 m}$, where $\hat{\phi}^{j}$ means that the form is omitted. Then we have

$$
\begin{aligned}
d\left(\phi^{1} \wedge \cdots \wedge \hat{\phi}^{j} \wedge \cdots \wedge \phi^{2 m}\right) & \\
= & \sum_{k<j}(-1)^{k+1} d \phi^{k} \wedge \phi^{1} \wedge \cdots \wedge \hat{\phi}^{k} \wedge \cdots \wedge \hat{\phi}^{j} \wedge \cdots \wedge \phi^{2 m} \\
& +\sum_{k>j}(-1)^{k} d \phi^{k} \wedge \phi^{1} \wedge \cdots \wedge \hat{\phi}^{j} \wedge \cdots \wedge \hat{\phi}^{k} \wedge \cdots \wedge \phi^{2 m} \\
= & \sum_{k<j}(-1)^{k}\left(\sum_{l<n} C_{l n}^{k} \phi^{l} \wedge \phi^{n}\right) \wedge \phi^{1} \wedge \cdots \wedge \hat{\phi}^{k} \wedge \cdots \wedge \hat{\phi}^{j} \wedge \cdots \wedge \phi^{2 m} \\
& +\sum_{k>j}(-1)^{k+1}\left(\sum_{l<n} C_{l n}^{k} \phi^{l} \wedge \phi^{n}\right) \wedge \phi^{1} \wedge \cdots \wedge \hat{\phi}^{j} \wedge \cdots \wedge \hat{\phi}^{k} \wedge \cdots \wedge \phi^{2 m} \\
= & \sum_{k<j}(-1)^{k} C_{k j}^{k} \phi^{k} \wedge \phi^{j} \wedge \phi^{1} \wedge \cdots \wedge \hat{\phi}^{k} \wedge \cdots \wedge \hat{\phi}^{j} \wedge \cdots \wedge \phi^{2 m} \\
& +\sum_{k>j}(-1)^{k+1} C_{j k}^{k} \phi^{j} \wedge \phi^{k} \wedge \phi^{1} \wedge \cdots \wedge \hat{\phi}^{j} \wedge \cdots \wedge \hat{\phi}^{k} \wedge \cdots \wedge \phi^{2 m} \\
= & (-1)^{j} \sum_{k} C_{j k}^{k} V o l=(-1)^{j} \operatorname{Tr}^{k}\left(a d_{e_{j}}\right) V o l,
\end{aligned}
$$

where $V o l=\phi^{1} \wedge \cdots \wedge \phi^{2 m}$, and the last equality follows by definition of trace

$$
\operatorname{Tr}\left(a d_{e_{j}}\right)=\sum_{k}\left\langle a d_{e_{j}}\left(e_{k}\right), e_{k}\right\rangle=\sum_{k} C_{j k}^{k} .
$$

If $\mathfrak{g}$ is unimodular, the trace of the adjoint vanishes, and so does the differential.

We are ready to proceed with the proof.

Proof of Theorem 6.1 Since $G$ is a connected, simply connected solvable Lie Group that admits a lattice, it is unimodular and by Lemma 6.4, condition (6.10) is satisfied.

Proposition 6.2 and 6.3 are valid for $\mathfrak{g}$ and so the left-invariant Dolbeault cohomology group of $M$ are isomorphic to left-invariant $\bar{\partial} \bar{\mu}$-harmonic forms and satisfy Serre duality. This proves (6.7), and also (6.8) for the first page.

For $r=1$, note that $\left({ }^{L} E_{1}^{*, *}, d_{1}\right)$ satisfies the hypothesis of the main theorem in [15]. In fact we proved that $d$ vanishes on $A_{\mathfrak{g}^{\mathbb{C}}}^{2 m-1}$, thus also $\partial, \bar{\partial}$ and $\bar{\mu}$ vanish on $A_{\mathfrak{g}^{\mathbb{C}}}^{2 m-1}$. $d_{1}$ is a sum of such differentials, thus $d_{1}=0$. Serre duality at first page, $d_{1}=0$ on $A_{\mathfrak{g}^{\mathbb{C}}}^{2 m-1}$ and ${ }^{L} E_{1}^{m, m} \cong \mathbb{C}$ imply Serre duality at every page. 
We conclude the section applying our results to solvmanifolds and to compact Lie Groups.

Proposition 6.5 Let $M=\Gamma \backslash G$ be a solvmanifold endowed with a left-invariant almost complex structure $J$. Denote by ${ }^{L} H_{\mathbb{C}}^{+}$, respectively ${ }^{L} H_{D o l}^{p, q}$, its left-invariant $J$-invariant cohomology, respectively its left-invariant Dolbeault cohomology, and by $H_{\mathbb{C}}^{+}$, respectively $H_{D o l}^{p, q}$, the non-left-invariant cohomologies.

Assume that ${ }^{L} H_{\mathbb{C}}^{+} \nsubseteq{ }^{L} H_{\text {Dol }}^{1,1}$. Then $H_{\mathbb{C}}^{+} \nsubseteq H_{\text {Dol }}^{1,1}$.

Proof Consider the following maps, induced in cohomology by the identity:

$$
\begin{aligned}
& { }^{L} H_{\mathbb{C}}^{+} \longrightarrow H_{\mathbb{C}}^{+} \\
& { }^{L}\left[\alpha^{1,1}\right]^{+} \longmapsto\left[\alpha^{1,1}\right]^{+}
\end{aligned}
$$

and

$$
\begin{aligned}
& { }^{{ }^{L}} H_{\text {Dol }}^{1,1} \longrightarrow H_{\text {Dol }}^{1,1} \\
& { }^{L}\left[\alpha^{1,1}\right]_{\text {Dol }} \longmapsto\left[\alpha^{1,1}\right]_{\text {Dol }}
\end{aligned}
$$

They are well defined since left-invariant forms are also ordinary differential forms, and they are injective because of the equalities

$$
\begin{aligned}
& { }^{L} H_{\mathbb{C}}^{+} \cap[0]^{+}={ }^{L}[0]^{+} \\
& { }^{L} H_{D o l}^{1,1} \cap[0]_{D o l}={ }^{L}[0]_{D o l}
\end{aligned}
$$

These equalities follow from the commutativity of the differential $d$ with the operator $L_{\gamma}^{*}$, i.e. the pullback of left multiplication by an element $\gamma \in \Gamma$. In fact commutativity of the operators implies that left-invariant forms that are the differential of a form, are actually the differential of a left-invariant form, and this proves (6.14). Separating the bidegree of the components of $d$, we have that

$$
\left[\bar{\mu}, L_{\gamma}^{*}\right]=\left[\bar{\partial}, L_{\gamma}^{*}\right]=0
$$

and this proves (6.15). This gives an injection of left-invariant cohomology groups into the non-left-invariant ones. The same reasoning shows that analogous monomorphisms are present for the groups $E_{1}^{0,1}, E_{2}^{0,1}$ and their left-invariant version, since $E_{2}^{0,1}$ is computed as the kernel of $d_{1}$, that again commutes with $L_{\gamma}^{*}$.

Theorem 4.2 is true at level of non-left-invariant cohomology, and of course also at level of left-invariant cohomologies thanks to the injection.

Assume now that ${ }^{L} H_{\mathbb{C}}^{+} \nsubseteq{ }^{L} H_{D o l}^{1,1}$. As a consequence of Theorem $4.2,{ }^{L} E_{1}^{0,1} \nsucceq$ ${ }^{L} E_{2}^{0,1}$. On the other side, if $E_{1}^{0,1} \cong E_{2}^{0,1}$, then ${ }^{L} E_{1}^{0,1} \cong{ }^{L} E_{2}^{0,1}$ at the level of leftinvariant cohomology. In particular, ${ }^{L} E_{1}^{0,1} \nsucceq{ }^{L} E_{2}^{0,1}$ implies $E_{1}^{0,1} \nsucceq E_{2}^{0,1}$, and using again Theorem 4.2, we conclude that $H_{\mathbb{C}}^{+} \nsubseteq H_{D o l}^{1,1}$. 
This proposition allows to use a property easy to check on left-invariant cohomology to recover information on non-left-invariant cohomology. An analogous result can be stated for compact Lie Groups.

Let $G$ be a compact Lie Group endowed with a left-invariant almost complex structure $J$, and $\mathfrak{g}$ its Lie Algebra.

The left-invariant Dolbeault cohomology of $G$ is

$$
{ }^{L} H_{D o l}^{p, q}(G)=H_{D o l}^{p, q}(\mathfrak{g}, J) .
$$

In the same way, the left-invariant spectral sequence is given by

$$
{ }^{L} E_{r}^{p, q}(G)=E_{r}^{p, q}(\mathfrak{g}, J) .
$$

We recall here a result from Cirici and Wilson that shows how it is possible to inject the left-invariant spectral sequence into the non-left-invariant one.

Proposition 6.6 ([6], Lemma 5.9) Let $G$ be a compact Lie Group with a left-invariant almost complex structure $J$. For all $r \geq 0$, the inclusion ${ }^{L} A^{*}(G) \hookrightarrow A^{*}(G)$ induces an injection

$$
{ }^{L} E_{r}^{p, q}(G) \hookrightarrow E_{r}^{p, q}(G, J) .
$$

As an immediate consequence of Theorem 4.2 and Proposition 6.6, we can deduce the following proposition for compact Lie Groups.

Proposition 6.7 Let $G$ be a compact Lie Group endowed with a left-invariant almost complex structure J. Assume that ${ }^{L} H_{\mathbb{C}}^{+} \nsubseteq{ }^{L} H_{\text {Dol }}^{1,1}(G)$. Then $H_{\mathbb{C}}^{+} \nsubseteq H_{\text {Dol }}^{1,1}$.

\section{Examples}

In this section we show some example of what was proved in Sect. 4 and in Sect. 6.

We begin showing that, on a complex manifold, $(*)$ is not a closed condition. We also explictly show with computations that when it is not satisfied, cohomology classes in $H_{\mathbb{C}}^{+}$do not define cohomology classes in $H_{\bar{\partial}}^{1,1}$.

Example 7.1 $\left(E_{1}^{0,1} \cong E_{2}^{0,1}\right.$ is not a closed condition) We provide two examples of not closedness of condition

$$
E_{1}^{0,1} \cong E_{2}^{0,1}
$$

The first one has appeared in [5], Example 4.8, with the purpose of showing different behaviours for the spectral sequence of a 6-dimensional nilmanifold. The example exhibits a curve of complex structures parametrized by $t$, in order to prove that the numbers $\operatorname{dim} E_{2}^{p, q}(t)$ are neither upper semi-continuos nor lower semi-continuous with respect to $t$. The spectral sequence behaves as follows:

- For $\sin t=1$ we have $E_{1}^{\bullet, \bullet} ¥ E_{2}^{\bullet, \bullet} ¥ E_{3}^{\bullet, \bullet} \cong E_{\infty}^{\bullet \bullet \bullet}$; 
- For $\sin t=-1$ we have $E_{1}^{\bullet, \bullet} ¥ E_{2}^{\bullet, \bullet} \cong E_{\infty}^{\bullet, \bullet}$;

- For $|\sin t| \neq 1$ we have $E_{1}^{\bullet, \bullet} \cong E_{2}^{\bullet, \bullet} \nsucceq E_{3}^{\bullet, \bullet} \cong E_{\infty}^{\bullet, \bullet}$;

We recall here the construction of the complex structure. Consider a 6-dimensional real Lie Algebra spanned by $\left\{e_{j}\right\}, j=1, \ldots, 6$. The differentials of the dual basis determine the Lie Algebra $\mathfrak{g}$ and we take them to be as follows:

$d e^{1}=0, \quad d e^{2}=0, \quad d e^{3}=0, \quad d e^{4}=e^{12}, \quad d e^{5}=e^{13}+e^{42}, \quad d e^{6}=e^{14}+e^{23}$.

Consider the family of complex structure parametrized by $t$, given by

$$
\begin{aligned}
& J_{t} e^{1}=-\sqrt{\frac{3(3-\sin t)(7+3 \sin t)}{(5+\sin t)(11-\sin t)}} e^{2}, \\
& J_{t} e^{3}=\sqrt{\frac{3(3-\sin t)(11-\sin t)}{(5+\sin t)(7+3 \sin t)}} e^{4}, \\
& J_{t} e^{5}=-\sqrt{\frac{(11-\sin t)(7+3 \sin t)}{3(3-\sin t)(5+\sin t)}} e^{6},
\end{aligned}
$$

and the nilpotent complex Lie Algebra obtained as

$$
\begin{aligned}
& 4 \varphi_{t}^{1}=\sqrt{(11-\sin t)(5+\sin t)} e^{1}+i \sqrt{3(3-\sin t)(7+3 \sin t)} e^{2} \\
& 8 \varphi_{t}^{2}=(5+\sin t)(7+3 \sin t) e^{3}-i \sqrt{3(5+\sin t)(3-\sin t)(11-\sin t)(7+3 \sin t)} e^{4}
\end{aligned}
$$

and

$$
\begin{aligned}
128 \varphi_{t}^{3}= & (5+\sin t)(7+3 \sin t)\left[3(3-\sin t) \sqrt{(11-\sin t)(5+\sin t)} e^{5}\right. \\
& \left.+i(11-\sin t) \sqrt{3(3-\sin t)(7+3 \sin t)} e^{6}\right]
\end{aligned}
$$

The basis of $(1,0)$-forms parametrized by $t$, has the following differentials:

$$
d \varphi_{t}^{1}=0, \quad d \varphi_{t}^{2}=\varphi_{t}^{1 \overline{1}}, \quad d \varphi_{t}^{3}=\frac{1-\sin t}{2} \varphi_{t}^{12}+2 \varphi_{t}^{1 \overline{2}}+\frac{1+\sin t}{4} \varphi_{t}^{2 \overline{1}}
$$

If $|\sin t| \neq 1$, we have that the first page of the spectral sequence is isomorphic, as a vector space, to the second one (but not to the third one). In particular, condition $(*)$ is satisfied. For $\sin t=1$, we have

$$
d \varphi_{t}^{1}=0, \quad d \varphi_{t}^{2}=\varphi_{t}^{1 \overline{1}}, \quad d \varphi_{t}^{3}=\varphi_{t}^{1 \overline{2}}+\frac{1}{2} \varphi_{t}^{2 \overline{1}}
$$

thus $H_{\bar{\partial}}^{0,1} \cong \mathbb{C}^{3}$, while $E_{2}^{0,1} \cong \mathbb{C}^{2}$ and $(*)$ is not satisfied.

More explictly, $d \varphi_{t}^{\overline{3}}=\partial \varphi_{t}^{\overline{3}}=\varphi_{t}^{\overline{1} 2}+\frac{1}{2} \varphi_{t}^{\overline{2} 1}$ is a $d$-exact 2-form and thus belongs to the 0 class in $H_{\mathbb{C}}^{+}$. However, it does not belong to the 0 class in the Dolbeault cohomology 
of $(1,1)$-forms since it is not the $\bar{\partial}$ of any $(1,0)$-form, and so the inclusion $H_{\mathbb{C}}^{+} \subseteq H_{\bar{\partial}}^{1,1}$ is not well defined.

The second example comes from the holomorphically parallelizable Nakamura manifold, and its small deformations. The manifold can be obtained as the quotient of the Lie Group $G=\mathbb{C} \ltimes_{\phi} \mathbb{C}^{2}$, with

$$
\phi(z)=\left[\begin{array}{cc}
e^{z} & 0 \\
0 & e^{-z}
\end{array}\right]
$$

by the lattice $\Gamma=\langle a+i b, c+i d\rangle$. If we assume that $b, d \in \pi \mathbb{Z}$ (cf. [1], Example 3.4), we can compute the Dolbeault cohomology of the manifold using a sub-complex of differential forms spanned by the $(0,1)$-forms

$$
d z^{\overline{1}}, \quad e^{-z_{1}} d z^{\overline{2}}, \quad e^{z_{1}} d z^{\overline{3}}
$$

and the conjugate $(1,0)$-forms. In [2], it is proved that we can compute the Dolbeault cohomology of a holomorphically parallelizable solvmanifold and its small deformations along special curves by means of a suitable sub-complex of differential forms (cf. [2], Corollary 3.7). In particular, for the Nakamura manifold, one can consider a curve of complex strcuture given by

$$
t d z^{\overline{1}} \otimes \frac{\partial}{\partial z_{1}} .
$$

By [2], Proposition 4.2, along such a curve and for all $t \neq 0$, the $\partial \bar{\partial}$-lemma is satisfied, the spectral sequence of deformations of the Nakamura manifold degenerates at the first page, and so condition $(*)$ is satisfied. On the contrary, for $t=0$ the spectral sequence of the holomorphically parallelizable Nakamura manifold does not degenerate at the first page.

The above manifolds, both provide a proof of Proposition 4.3.

We proceed now to compute the Dolbeault left-invariant cohomology of 4dimensional solvmanifolds that do not admit integrable almost complex structures. There exists three such solvmanifolds (cf. [4]). The first one is a nilmanifold. The second one is a symplectic, completely solvable but not nilpotent solvmanifold (Example 7.2) and the last one is a completely solvable but not symplectic nor nilpotent solvmanifold (Example 7.5). Calculations for the nilmanifold have already been made in [6], Example 5.15.

Example 7.2 $\left(\Gamma \backslash \operatorname{Sol}(3) \times \mathbb{S}^{1}\right)$ Denote by Sol (3) the solvable Lie Group of dimension 3. It can be obtained considering the groups $(\mathbb{R},+),\left(\mathbb{R}^{2},+\right)$ and taking the semi-direct product $\operatorname{Sol}(3)=\mathbb{R} \ltimes_{\phi} \mathbb{R}^{2}$, with

$$
\phi(t)=\left[\begin{array}{cc}
e^{t} & 0 \\
0 & e^{-t}
\end{array}\right] .
$$


The product $\operatorname{Sol}(3) \times \mathbb{R}$ can be identified as a subgroup of matrices via the homomorphism

$$
(t, x, y, s) \stackrel{\theta}{\longmapsto}\left[\begin{array}{crrrr}
e^{t} & 0 & x & 0 & 0 \\
& e^{-t} & y & 0 & 0 \\
& & 1 & 0 & 0 \\
& & 1 & s \\
& & & 1
\end{array}\right] \in S L(5, \mathbb{R})
$$

Denote with $K$ the image of $\operatorname{Sol}(3) \times \mathbb{R}$ by $\theta$. Then $K$ is a subgroup of $\operatorname{SL}(5, \mathbb{R})$ with respect to matrix multiplication, isomorphic to $\operatorname{Sol}(3) \times \mathbb{R}$. By Auslander et al. [3] (Theorem 4), $K$ admits a lattice $\Gamma$. The quotient $M=\Gamma \backslash K$ is a solvmanifold, of real dimension 4. An explicit construction of $M$ can be found in [4] in the examples following the classification of four-dimensional solvmanifolds. Taking $A \in K$ and computing $A^{-1} d A$, we obtain a basis of left-invariant forms

$$
\left\{e^{1}=d t, e^{2}=e^{-t} d x, e^{3}=e^{t} d y, e^{4}=d s\right\}
$$

The dual basis of vector fields is

$$
\left\{e_{1}=\frac{\partial}{\partial t}, e_{2}=e^{t} \frac{\partial}{\partial x}, e_{3}=e^{-t} \frac{\partial}{\partial y}, e_{4}=\frac{\partial}{\partial s}\right\} .
$$

The only non-zero brackets are

$$
\left[e_{1}, e_{2}\right]=e_{2}, \quad\left[e_{1}, e_{3}\right]=-e_{3},
$$

and the differentials of 1 -forms can be obtained directly differentiating $\left\{e^{j}\right\}$, or by taking the dual of the Lie bracket. The only non-vanishing differentials are

$$
d e^{2}=-e^{12}, \quad d e^{3}=e^{13}, \quad d e^{24}=-e^{124}, \quad d e^{34}=e^{134} .
$$

We can directly compute the left-invariant de Rham cohomology of $M$ and, since $\operatorname{Sol}(3) \times \mathbb{R}$ is completely solvable, it coincides with the real de Rham Cohomology:

$$
\begin{aligned}
& H_{d R}^{0}(M ; \mathbb{R})=\langle 1\rangle, \\
& H_{d R}^{1}(M ; \mathbb{R})=\left\langle e^{1}, e^{4}\right\rangle, \\
& H_{d R}^{2}(M ; \mathbb{R})=\left\langle e^{14}, e^{23}\right\rangle, \\
& H_{d R}^{3}(M ; \mathbb{R})=\left\langle e^{123}, e^{234}\right\rangle, \\
& H_{d R}^{4}(M ; \mathbb{R})=\left\langle e^{1234}\right\rangle
\end{aligned}
$$

In particular, the Euler characteristic of $M$ is 0 . The complex de Rham cohomology of $M$ is obtained as the complexified of the real one. 
We shall consider two almost complex structures on $M$. Set
(A) $\left\{\begin{array}{l}J e_{1}=e_{2}, \\ J e_{3}=e_{4},\end{array}\right.$
(B) $\left\{\begin{array}{l}J e_{1}=e_{4}, \\ J e_{2}=e_{3} .\end{array}\right.$

Structure (A) will provide an example of a left-invariant spectral sequence degenerating at the first page. This almost complex strcuture is not almost-Kähler. On the other side, structure (B) will provide an example of a left-invariant spectral sequence degenerating at the second page, and it is an almost-Kähler structure.

Regarding structure (A), a basis of complex vector fields is obtained by taking projections of $e_{1}$ and $e_{3}$. Define

$$
Z_{1}=\pi^{1,0}\left(e_{1}\right)=\frac{1}{2}\left(e_{1}-i e_{2}\right), \quad Z_{2}=\pi^{1,0}\left(e_{3}\right)=\frac{1}{2}\left(e_{3}-i e_{4}\right) .
$$

A basis of the complexified tangent space is $\left\{Z_{1}, Z_{2}, \bar{Z}_{1}, \bar{Z}_{2}\right\}$, and a basis of dual forms for the complexified Lie Algebra is $\left\{\varphi^{1}, \varphi^{2}, \bar{\varphi}^{1}, \bar{\varphi}^{2}\right\}$, where

$$
\varphi^{1}=e^{1}+i e^{2}, \quad \varphi^{2}=e^{3}+i e^{4} .
$$

As for the de Rham cohomology, the differentials $\mu, \partial, \bar{\partial}, \bar{\mu}$ of complex forms can be obtained calculating the differential of the complex forms starting from the real ones, then separating the bidegrees, or else by duality from the brackets. The differentials are

$$
\begin{aligned}
1-\text { forms: } \quad \mu \varphi^{1} & =0, \quad \partial \varphi^{1}=0, \quad \bar{\partial} \varphi^{1}=\frac{1}{2} \varphi^{1 \overline{1}}, \quad \bar{\mu} \varphi^{1}=0, \\
\mu \varphi^{2} & =0, \quad \partial \varphi^{2}=\frac{1}{4} \varphi^{12}, \quad \bar{\partial} \varphi^{2}=\frac{1}{4}\left(\varphi^{1 \overline{2}}+\varphi^{\overline{1} 2}\right), \quad \bar{\mu} \varphi^{2}=\frac{1}{4} \varphi^{\overline{1} \overline{2}},
\end{aligned}
$$

and the conjugate equations. Note that the almost complex structure is not integrable since $\bar{\mu} \varphi^{2} \neq 0$. From the differentials on 1 -form, we can compute them for all degrees, and then compute the left-invariant spectral sequence. As a consequence of Theorem 6.1, it satisfies Serre duality. We show calculations only for bidegree $(1,1)$. On

$$
A_{\mathbb{C}}^{1,1}=\left\langle\varphi^{1 \overline{1}}, \varphi^{1 \overline{2}}, \varphi^{\overline{1} 2}, \varphi^{2 \overline{2}}\right\rangle
$$

$\bar{\mu}$ vanishes for bidegree reasons. $\bar{\partial}$ vanishes on $\varphi^{1 \overline{1}}$, and $\bar{\partial} \varphi^{1 \overline{2}}=\bar{\partial} \varphi^{\overline{1} 2}=\bar{\mu} \varphi^{12}$, while $\bar{\partial} \varphi^{2 \overline{2}}$ does not belong to the image of $\bar{\mu}$, then

$$
X_{1}^{1,1}=\left\langle\varphi^{1 \overline{1}}, \varphi^{1 \overline{2}}, \varphi^{\overline{1} 2}\right\rangle
$$

For bidegree reason, $(1,1)$-forms cannot be in the image of $\bar{\mu}$, so we just have to check the existence of a $(1,0)$-form $\beta^{1,0}$ such that $\eta^{1,1}=\bar{\partial} \beta^{1,0}$ and $\bar{\mu} \beta^{1,0}=0$. In general, $\beta^{1,0}=a \varphi^{1}+b \varphi^{2}$. The condition $\bar{\mu} \beta^{1,0}=0$ gives 


$$
\bar{\mu} \beta^{1,0}=\frac{b}{4} \varphi^{\overline{1} \overline{2}}=0,
$$

so it has to be $\beta^{1,0}=a \varphi^{1}$. Then

$$
\bar{\partial} \beta^{1,0}=\frac{a}{2} \varphi^{1 \overline{1}}
$$

so that $Y_{1}^{1,1}=\left\langle\varphi^{1 \overline{1}}\right\rangle$. Taking the quotient, we have

$$
{ }^{L} H_{D o l}^{1,1}(M)=\left\langle\varphi^{1 \overline{2}}, \varphi^{\overline{1} 2}\right\rangle \cong \mathbb{C}^{2}
$$

Analogous calculations show that

$$
\begin{aligned}
& { }^{L} H_{D o l}^{1,0}(M)={ }^{L} H_{D o l}^{2,0}(M)={ }^{L} H_{D o l}^{0,2}(M)={ }^{L} H_{D o l}^{1,2}(M)=\{0\}, \\
& { }^{L} H_{D o l}^{0,1}(M)=\left\langle\varphi^{\overline{1}}, \varphi^{\overline{2}}\right\rangle \cong \mathbb{C}^{2}, \\
& { }^{L} H_{D o l}^{2,1}(M)=\left\langle\varphi^{12 \overline{1}}, \varphi^{12 \overline{2}}\right\rangle \cong \mathbb{C}^{2}, \\
& { }^{L} H_{D o l}^{0,0}(M)=\langle 1\rangle \cong \mathbb{C}, \\
& { }^{L} H_{D o l}^{2,2}(M)=\left\langle\varphi^{12 \overline{1} \overline{2}}\right\rangle \cong \mathbb{C} .
\end{aligned}
$$

The first page is

$$
{ }_{A}^{L} E_{1}^{*, *} \cong \begin{array}{|c|c|c|}
\hline 0 & 0 & \mathbb{C} \\
\hline \mathbb{C}^{2} & \mathbb{C}^{2} & \mathbb{C}^{2} \\
\hline \mathbb{C} & 0 & 0 \\
\hline
\end{array}
$$

Note that the spectral sequence degenerates at the first page because separately in every bidegree, the dimension can only decrease and at the last page their sum must coincide with the Betti numbers of $M$. To compute the real $J$-invariant group $H^{+}$, we write forms in $H_{d R}^{2}$ as complex forms:

$$
\begin{aligned}
e^{14} & =\frac{\varphi^{1}+\varphi^{\overline{1}}}{2} \wedge \frac{\varphi^{2}-\varphi^{\overline{2}}}{2 i}=\frac{1}{4 i}\left(\varphi^{12}+\varphi^{\overline{1} 2}-\varphi^{1 \overline{2}}-\varphi^{\overline{1} \overline{2}}\right), \\
e^{23} & =\frac{\varphi^{1}-\varphi^{\overline{1}}}{2 i} \wedge \frac{\varphi^{2}+\varphi^{\overline{2}}}{2}=\frac{1}{4 i}\left(\varphi^{12}-\varphi^{\overline{1} 2}+\varphi^{1 \overline{2}}-\varphi^{\overline{1} \overline{2}}\right) .
\end{aligned}
$$

First adding then subtracting we obtain $e^{14}-e^{23} \in H^{+}$and $e^{14}+e^{23} \in H^{-}$,

$$
H^{+}=\left\langle\frac{1}{2 i}\left(\varphi^{\overline{1} 2}-\varphi^{1 \overline{2}}\right)\right\rangle, \quad H^{-}=\left\langle\frac{1}{2 i}\left(\varphi^{12}-\varphi^{\overline{1} \overline{2}}\right)\right\rangle .
$$


This is sufficient to conclude the computation of $\mathrm{H}^{+}$and $\mathrm{H}^{-}$since in dimension 4, we always have a direct sum decomposition

$$
H_{d R}^{2}(M ; \mathbb{R})=H^{+} \oplus H^{-},
$$

(cf. [10], Theorem 2.3).

For structure (B), the $d$-closed 2-form providing an almost-Kähler metric is $\omega=$ $e^{14}+e^{23}$. The computations of the left-invariant spectral sequence proceed in the same way as for structure (A). The only non-vanishing differentials are

$$
\bar{\partial} \varphi^{2}=-\frac{1}{2} \varphi^{1 \overline{2}}, \quad \bar{\mu} \varphi^{2}=-\frac{1}{2} \varphi^{\overline{1} \overline{2}}, \quad \bar{\mu} \varphi^{12}=\frac{1}{2} \varphi^{1 \overline{1} \overline{2}}, \quad \partial \varphi^{1 \overline{2}}=-\frac{1}{2} \varphi^{12 \overline{1}},
$$

and the conjugate equations. The computation of Dolbeault cohomology group is straightforward:

$$
\begin{aligned}
& { }^{L} H_{D o l}^{0,0}(M)=\langle 1\rangle, \quad{ }^{L} H_{D o l}^{2,2}(M)=\left\langle\varphi^{12 \overline{1} \overline{2}}\right\rangle, \\
& { }^{L} H_{D o l}^{1,1}(M)=\left\langle\varphi^{1 \overline{1}}, \varphi^{2 \overline{2}}, \varphi^{1 \overline{2}}, \varphi^{\overline{1} 2}\right\rangle \cong \mathbb{C}^{4}, \\
& { }^{L} H_{D o l}^{0,1}(M)=\left\langle\varphi^{\overline{1}}, \varphi^{\overline{2}}\right\rangle, \quad{ }^{L} H_{D o l}^{2,1}(M)=\left\langle\varphi^{12 \overline{1}}, \varphi^{12 \overline{2}}\right\rangle, \\
& { }^{L} H_{D o l}^{1,0}(M)=\left\langle\varphi^{1}\right\rangle, \quad{ }^{L} H_{D o l}^{1,2}(M)=\left\langle\varphi^{2 \overline{1} \overline{2}}\right\rangle,
\end{aligned}
$$

and the first page of the spectral sequence is

$$
{ }_{B}^{L} E_{1}^{*, *} \cong \begin{array}{|c|c|c|}
\hline 0 & \mathbb{C} & \mathbb{C} \\
\hline \mathbb{C}^{2} & \mathbb{C}^{4} & \mathbb{C}^{2} \\
\hline \mathbb{C} & \mathbb{C} & 0 \\
\hline
\end{array}
$$

Note that this is not the bigrading induced on the complex de Rham cohomology, since for example $\operatorname{dim}{ }^{L} H_{D o l}^{1,0}+\operatorname{dim}{ }^{L} H_{D o l}^{0,1} \supsetneqq \operatorname{dim} H_{d R}^{1}$. The same happens for degree 2 and 3. The following page is obtained as $E_{2}^{p, q} \cong X_{2}^{p, q} / Y_{2}^{p, q}$. The quotients are

$$
\begin{aligned}
& { }^{{ }^{L}} E_{2}^{0,0}=\langle 1\rangle, \quad{ }^{L} E_{2}^{2,2}=\left\langle\varphi^{12 \overline{1} \overline{2}}\right\rangle, \\
& { }^{L} E_{2}^{1,0}=\left\langle\varphi^{1}\right\rangle, \quad{ }^{L} E_{2}^{1,2}=\left\langle\varphi^{2 \overline{1} \overline{2}}\right\rangle, \\
& { }^{L} E_{2}^{0,1}=\left\langle\varphi^{\overline{1}}\right\rangle, \quad{ }^{L} E_{2}^{2,1}=\left\langle\varphi^{12 \overline{2}}\right\rangle, \\
& { }^{L} E_{2}^{2,0}={ }^{L} E_{2}^{0,2}=\{0\}, \\
& { }^{L} E_{2}^{1,1}=\left\langle\varphi^{1 \overline{1}}, \varphi^{2 \overline{2}}\right\rangle .
\end{aligned}
$$

For dimension reasons, this is also the $\infty$ page of the spectral sequence,

$$
{ }_{B}^{L} E_{r}^{*, *} \cong \begin{array}{|l|l|l|}
\hline 0 & \mathbb{C} & \mathbb{C} \\
\hline \mathbb{C} & \mathbb{C} & \mathbb{C} \\
\hline \mathbb{C} & \mathbb{C} & 0 \\
\hline
\end{array}, \quad \forall r \geq 2,
$$


and gives the induced bigrading on the de Rham cohomology. Proceeding as for structure (A),

$$
H^{+}=\left\langle i \varphi^{1 \overline{1}}, i \varphi^{2 \overline{2}}\right\rangle, \quad H^{-}=\{0\} .
$$

Remark 7.3 In both examples, harmonic representatives of $H_{\mathbb{C}}^{+}$are also harmonic representative of ${ }^{L} H_{D o l}^{1,1}$. For structure (A), the condition ${ }^{L} E_{1}^{0,1} \cong{ }^{L} E_{2}^{0,1}$ is satisfied, and if we consider a non-harmonic representative in $H_{\mathbb{C}}^{+}$, it still defines a class in ${ }^{L} H_{D o l}^{1,1}$, as expected from Theorem 4.2. In fact we have $H_{\mathbb{C}}^{+}=\left\langle\varphi^{\overline{1} 2}-\varphi^{1 \overline{2}}\right\rangle$. The generator is $d$-closed. $d$-exact $(1,1)$ forms are written as $\bar{\partial} \beta^{1,0}+\partial \beta^{0,1}$, with

$$
\beta^{1,0}=a \varphi^{1}+b \varphi^{2}, \quad \beta^{0,1}=c \varphi^{\overline{1}}+d \varphi^{\overline{2}}
$$

with the conditions $\bar{\mu} \beta^{1,0}+\bar{\partial} \beta^{0,1}=0$ and $\mu \beta^{0,1}+\partial \beta^{1,0}=0$ that are satisfied only if $b+d=0$. Immediately we have

$$
\bar{\partial} \beta^{1,0}+\partial \beta^{0,1}=\frac{a-c}{2} \varphi^{1 \overline{1}}
$$

that is the 0 class in ${ }^{L} H_{D o l}^{1,1}$.

This is not true for structure (B), in fact if we modify $\varphi^{1 \overline{1}}$ with a $d$-exact $(1,1)$-form, the class in ${ }^{L} H_{D o l}^{1,1}$ varies.

Example 7.4 (Let-invariant deformations of $\left.\Gamma \backslash \operatorname{Sol}(3) \times \mathbb{S}^{1}\right)$ In this example we compute left-invariant deformations of the manifold $M=\Gamma \backslash \operatorname{Sol}(3) \times \mathbb{S}^{1}$. Consider the almost complex solvmanifold endowed with a left-invariant almost complex structure $(M, J)$, where $J$ is the structure (B) of Example 7.2. We study the behaviour of the left-invariant spectral sequence under deformations.

Since we are interested in the left-invariant cohomology, calculating deformations of $J$ is a matter of linear algebra. As a matrix, $J$ is written as

$$
J=\left[\begin{array}{cc}
0 & -I d_{2} \\
I d_{2} & 0
\end{array}\right]
$$

and its small deformations are represented by a $4 \times 4$ matrix $L$ satisfying

$$
L J+J L=0 .
$$

This last condition is written as

$$
L=\left[\begin{array}{cc}
A & B \\
P B P & -P A P
\end{array}\right]
$$

where $A$ and $B$ are $2 \times 2$ matrices and $P=\left[\begin{array}{ll}0 & 1 \\ 1 & 0\end{array}\right]$. 
The deformations are also codified by a form $\psi \in T^{1,0} M \otimes T^{0,1} M^{*}$ that is written as

$$
\psi=\psi_{1}^{1} \varphi^{\overline{1}} \otimes Z_{1}+\psi_{1}^{2} \varphi^{\overline{1}} \otimes Z_{2}+\psi_{2}^{1} \varphi^{\overline{2}} \otimes Z_{1}+\psi_{2}^{2} \varphi^{\overline{2}} \otimes Z_{2}
$$

and must satisfy $\psi=\frac{1}{2}(L-i J L)$. By writing out both members of the equality we obtain the expression of $\psi$ in function of $A$ and $B$, then we compute the brackets of the deformed structures in function of $\psi$ and the brackets at time 0 . Finally by duality we obtain the differentials of the deformed left-invariant forms, and compute the left-invariant spectral sequence. We classify deformations into two groups:

(i) $A_{21}+A_{12}=0$ and $B_{11}=0$,

(ii) the remaining structures.

For structures of type (i), the behaviour of the spectral sequence stays the same, and we have degeneracy at the second page. For structures of type (ii) and $t \neq 0$, we have that the spectral sequence degenerates at the first page and coincides with the spectral sequence of structure (A).

In particular this shows that condition ${ }^{L} E_{1}^{0,1}={ }^{L} E_{2}^{0,1}$ is not closed (at level of left-invariant spectral sequence), since it is not satisfied for $t=0$, but it is true for deformations of class $(i i)$.

Example 7.5 $(\Gamma \backslash G)$ Let $G=\mathbb{R} \ltimes_{\sigma} \mathbb{R}^{3}$, with

$$
\sigma(t)=\left[\begin{array}{ccc}
e^{\alpha_{2} t} & 0 & 0 \\
& e^{\alpha_{3} t} & 0 \\
& & e^{\alpha_{4} t}
\end{array}\right]
$$

with $\alpha_{j}$ real numbers satisfying $\alpha_{2}+\alpha_{3}+\alpha_{4}=0$. $G$ identifies as a subgroup of matrices, still denoted by $G$, via the homomorphism

$$
(t, x, y, z) \longmapsto\left[\begin{array}{cccc}
e^{\alpha_{2} t} & 0 & 0 & x \\
& e^{\alpha_{3} t} & 0 & y \\
& & e^{\alpha_{4} t} & z \\
& & & 1
\end{array}\right] \in S L(4, \mathbb{R})
$$

A proof that $G$ admits a lattice $\Gamma$, and an explicit construction of the quotient can be found, as for Example 7.2, in [4]. Then the quotient $M=\Gamma \backslash G$ is a solvmanifold, of real dimension 4. A basis of left-invariant forms is

$$
\left\{e^{1}=d t, e^{2}=e^{-\alpha_{2} t} d x, e^{3}=e^{-\alpha_{3} t} d y, e^{4}=e^{-\alpha_{4} t} d z\right\}
$$

The dual basis of vector fields is

$$
\left\{e_{1}=\frac{\partial}{\partial t}, e_{2}=e^{\alpha_{1} t} \frac{\partial}{\partial x}, e_{3}=e^{\alpha_{3} t} \frac{\partial}{\partial y}, e_{4}=e^{\alpha_{4} t} \frac{\partial}{\partial z}\right\}
$$


The only non-zero brackets are

$$
\left[e_{1}, e_{j}\right]=\alpha_{j} e_{j}, \quad j=2,3,4
$$

The non-vanishing differentials are

$$
\begin{aligned}
& d e^{2}=-\alpha_{2} e^{12}, \quad d e^{3}=-\alpha_{3} e^{13}, \quad d e^{4}=-\alpha_{4} e^{14}, \\
& d e^{23}=\alpha_{4} e^{123}, \quad d e^{24}=\alpha_{3} e^{124}, \quad d e^{34}=\alpha_{2} e^{134} \text {. }
\end{aligned}
$$

$G$ is completely solvable, and its left-invariant cohomology coincides with the real de Rham Cohomology:

$$
\begin{aligned}
& H_{d R}^{0}(M ; \mathbb{R})=\langle 1\rangle, \\
& H_{d R}^{1}(M ; \mathbb{R})=\left\langle e^{1}\right\rangle, \\
& H_{d R}^{2}(M ; \mathbb{R})=\{0\}, \\
& H_{d R}^{3}(M ; \mathbb{R})=\left\langle e^{234}\right\rangle, \\
& H_{d R}^{4}(M ; \mathbb{R})=\left\langle e^{1234}\right\rangle .
\end{aligned}
$$

We compute the left-invariant spectral sequence for the almost complex structure

$$
\left\{\begin{array}{l}
J e_{1}=e_{2}, \\
J e_{3}=e_{4},
\end{array}\right.
$$

The non-vanishing differentials on 1-forms are

$$
\begin{aligned}
& \bar{\partial} \varphi^{1}=\frac{\alpha_{2}}{2} \varphi^{1 \overline{1}}, \quad \partial \varphi^{2}=-\frac{\alpha_{3}+\alpha_{4}}{4} \varphi^{12}, \quad \bar{\mu} \varphi^{2}=-\frac{\alpha_{3}-\alpha_{4}}{4} \varphi^{\overline{1} \overline{2}}, \\
& \bar{\partial} \varphi^{2}=-\frac{\alpha_{3}+\alpha_{4}}{4} \varphi^{\overline{1} 2}-\frac{\alpha_{3}-\alpha_{4}}{4} \varphi^{1 \overline{2}} .
\end{aligned}
$$

The left-invariant Dolbeault cohomology is

$$
{ }^{L_{1}} E_{1}^{*, *} \cong \begin{array}{|c|c|c|}
\hline 0 & 0 & \mathbb{C} \\
\hline \mathbb{C}^{2} & \mathbb{C}^{2} & \mathbb{C}^{2} \\
\hline \mathbb{C} & 0 & 0 \\
\hline
\end{array}
$$

The spectral sequence degenerates at the second page, giving the bigrading of the de Rham cohomology:

$$
{ }^{L} E_{r}^{*, *} \cong \begin{array}{|l|l|l|}
0 & 0 & \mathbb{C} \\
\hline \mathbb{C} & 0 & \mathbb{C} \\
\hline \mathbb{C} & 0 & 0 \\
\hline
\end{array}, \quad \forall r \geq 2
$$


Acknowledgements The authors would like to thank Joana Cirici and Weiyi Zhang for useful comments and remarks. The authors also wish to thank the anonymous referee for the useful and precious suggestions, which led to a substantial improvement of some of the results presented in the paper.

Funding Open access funding provided by Università degli Studi di Parma within the CRUI-CARE Agreement.

Open Access This article is licensed under a Creative Commons Attribution 4.0 International License, which permits use, sharing, adaptation, distribution and reproduction in any medium or format, as long as you give appropriate credit to the original author(s) and the source, provide a link to the Creative Commons licence, and indicate if changes were made. The images or other third party material in this article are included in the article's Creative Commons licence, unless indicated otherwise in a credit line to the material. If material is not included in the article's Creative Commons licence and your intended use is not permitted by statutory regulation or exceeds the permitted use, you will need to obtain permission directly from the copyright holder. To view a copy of this licence, visit http://creativecommons.org/licenses/by/4.0/.

\section{References}

1. Angella, D., Kasuya, H.: Bott-Chern cohomology of solvmanifolds. Ann. Glob. Anal. Geom. 52(4), 363-411 (2017)

2. Angella, D., Kasuya, H.: Cohomologies of deformations of solvmanifolds and closedness of some properties. North-West. Eur. J. Math. 3, 75-105 (2017)

3. Auslander, L., Green, L., Hahn, F.: Flows on some three dimensional homogeneous spaces. Bull. Am. Math. Soc. 67(5), 494-497 (1961)

4. Bock, C.: On low-dimensional solvmanifolds. Asian J. Math. 20(2), 199-262 (2016)

5. Ceballos, M., Otal, A., Ugarte, L., Villacampa, R.: Invariant complex structures on 6-nilmanifolds: classification, Frölicher spectral sequence and special Hermitian metrics. J. Geom. Anal. 26, 252-286 (2016)

6. Cirici, J., Wilson, S.O.: Dolbeault cohomology for almost complex manifolds. Adv. Math. (to appear). arXiv: $1809.01416 \mathrm{v} 2$

7. Cirici, J., Wilson, S.O.: Topological and geometric aspects of almost Kähler manifolds via harmonic theory. Sel. Math. New Ser. 26(3), 35 (2020)

8. Coelho, R., Placini, G., Stelzig, J.: Maximally non-integrable almost complex structures: an $h$-principle and cohomological properties (2021). arXiv:2105.12113v2

9. Cordero, L.A., Fernandéz, M., Gray, A., Ugarte, L.: A general description of the terms in the Frölicher spectral sequence. Differ. Geom. Appl. 7(1), 75-84 (1997)

10. Drăghici, T., Li, T.-J., Zhang, W.: Symplectic forms and cohomology decomposition of almost complex four-manifolds. Int. Math. Res. Not. 2010, 1-17 (2010)

11. Drăghici, T., Li, T.-J., Zhang, W.: On the $J$-anti-invariant cohomology of almost complex 4-manifolds. Q. J. Math. 64, 83-111 (2013)

12. Frölicher, A.: Relations between the cohomology groups of Dolbeault and topological invariants. Proc. Natl. Acad. Sci. USA 41, 641-644 (1955)

13. Li, T.-J., Zhang, W.: Comparing tamed and compatible symplectic cones and cohomological properties of almost complex manifolds. Commun. Anal. Geom. 17, 651-683 (2009)

14. Livernet, M., Whitehouse, S., Ziegenhagen, S.: On the spectral sequence associated to a multicomplex. J. Pure Appl. Algebra 224(2), 528-535 (2020)

15. Milivojević, A.: Another proof of the persistence of Serre symmetry in the Frölicher spectral sequence. Complex Manifolds 7, 141-144 (2020)

16. Rollenske, S.: Dolbeault cohomology of nilmanifolds with left-invariant complex structure. Complex Differ. Geom., Springer Proc. Math. 8, 369-392 (2011)

17. Tardini, N., Tomassini, A.: Differential operators on almost-Hermitian manifolds and harmonic forms. Complex Manifolds 7, 106-128 (2020)

Publisher's Note Springer Nature remains neutral with regard to jurisdictional claims in published maps and institutional affiliations. 